\title{
A numerical study on cavity expansion in water: hydraulic ram under ballistic impacts
}

\author{
A. Charles ${ }^{1}$, E. Deletombe ${ }^{2} \&$ J. Dupas ${ }^{2}$ \\ ${ }^{I}$ CNRS - Laboratoire de Mécanique et d'Acoustique, UPR-705, France \\ ${ }^{2}$ Onera - The French Aerospace Lab, France
}

\begin{abstract}
In the field of aircraft safety, solving coupled high speed dynamic fluid-structure problems is sometimes needed, where large deformations or even rupture of the structure have to be considered. The presented work deals with the vulnerability of aircraft fuel tank to high velocity impacts, and more specifically with the prediction of hydraulic ram effects that such impacts can produce on fuel tank structures. In the present work, the authors explored the possibility offered by the EUROPLEXUS code to model the expansion and collapse of cavities that develop in containers after impact and arrest by water of ballistic projectiles. An original experimental test case is used to challenge the EUROPLEXUS code, which a $3 \mathrm{~kJ}, 850 \mathrm{~m} / \mathrm{s}, 7,72 \mathrm{~mm}$ projectile being fired in various containers, equipped with high speed cameras to capture cavity expansion and collapsing phases. The objective of the present numerical exercise was to evaluate which proportion of the kinetic energy of such a bullet could be converted into internal energy (pressure, heat), fluid kinetic energy and latent energy of the water, to estimate how much of, and how dynamically, this energy could finally be transferred to a deformable fuel tank structure to possibly damage it.

Keywords: hydraulic ram, FE simulations, cavity expansion.
\end{abstract}

\section{Introduction}

In the field of passive safety in aeronautics, solving fluid-structure coupling problems is a major field of research of the last decade. The main objective is to develop codes able to solve multi-physical problems in the frame of high-speed dynamics. The present work deals with the ballistic impact of a bullet in aircraft fuel tanks. The perforation of the tank is often unavoidable and induces a slow 
leak of fuel which allows the aircraft to return, but the possibility of global damage on the tank, of a major loss of the whole fuel, of a weakening of the structural strength and the destruction of the aircraft, has to be examined and prevented. Such global damage of the structure is due to the hydrodynamic effects that develop in the tank when the high velocity projectile passes through it. It is commonly referred to as Hydraulic Ram (HRAM) events in fuel tank structures.

The problem is quite complex and is still not very well modelled [1], all the more as experimental observation, analysis and validation remain very difficult. The physics engaged, when non academic high-speed deformable projectiles are considered, depends on so many and highly non-linear aspects (fluid, material, geometrical, etc), that no expert predictions or decisions can be made concerning the outcome of such HRAM scenarios without the help of costly numerical simulations. But the fluid-structure coupling (especially with the deformable projectile) generally leads to numerical instabilities. In the present paper, the EUROPLEXUS explicit research code was challenged to solve HRAM events especially the expansion and collapse of cavities that develop in containers after impact and arrest of ballistic projectiles by water. EUROPLEXUS is being developed together by the French CEA and EU CRC-ISPRA to study nuclear plants security questions. It is designed to accurately solve fast dynamic problems for coupled fluid/solid systems, and includes both finite element and volume element formulations to solve solid Lagrangian and fluid Eulerian interaction problems. To illustrate the problem, an original experimental test case is shown in fig. 1, with a subsonic $850 \mathrm{~m} / \mathrm{s}, 7,62 \mathrm{~mm}$ (3 kJ kinetic energy) projectile being fired in a large transparent water pool, equipped with high speed cameras to capture the $30 \mathrm{~ms}$ long cavity expansion and collapsing phases [2].

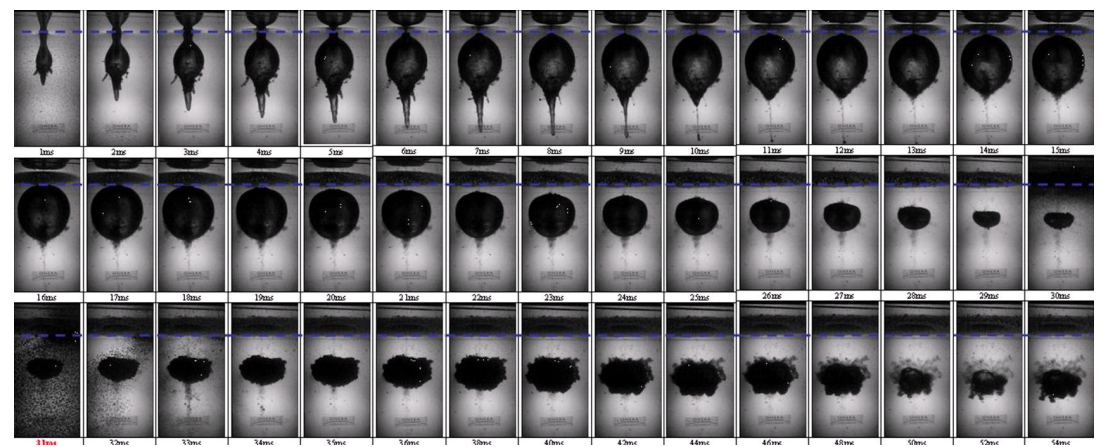

Figure 1: Cavity growth, decay and successive cycles in a large water pool (projectile trajectory from top to bottom).

Still, strong assumptions are made in the present work which greatly simplify the studied modelling problem. HRAM includes several stages, each one having its own time scale as presented in table 1 (see $[1,2]$ for a more detailed description of here studied HRAM events). One of our expectations was to 


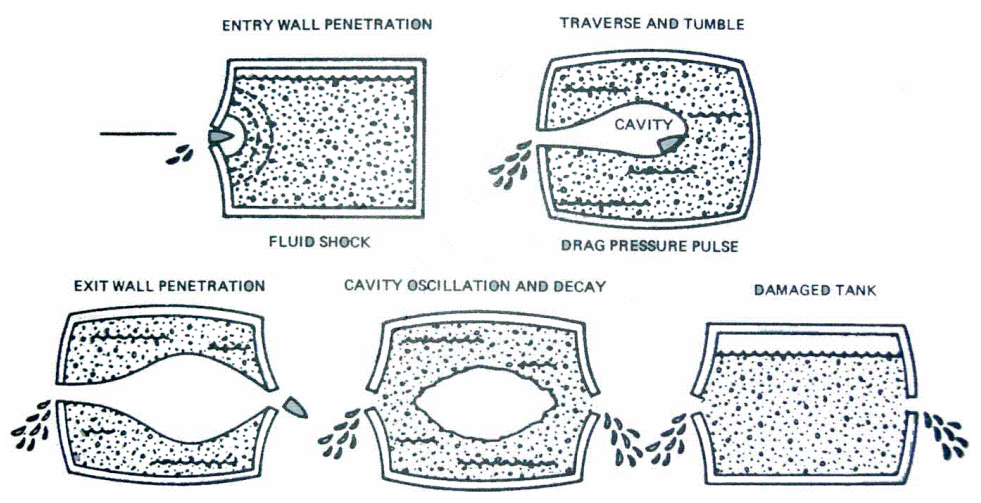

Figure 2: Successive stages of the hydrodynamic ram pressure (projectile trajectory from left to right) [3].

identify, if ever, the most or least determinant ones to be considered or disregarded when designing structural aircraft fuel tanks.

After the initial shock wave phase, as the subsonic projectile moves forward and tumbles through the liquid, it is submitted to high drag pressures that quickly slow it down. Its kinetic energy decreases and is transferred to the fluid as liquid kinetic energy, mechanical energy (pressure) and latent heat (cavitation). Most of the projectile kinetic energy is often lost before the shock wave has reflected back from the aircraft fuel tank walls, which means that the projectile kinematics is almost independent of the geometry of the container: the kinematics measured in the firing test depicted in fig. 1 is then considered as a reference one in the present work. Also, in the present paper, it was assumed that the pressure level that establishes in the cavity behind the projectile (see fig. 1), decreases down but not less - than the liquid saturation pressure, and that vaporisation of the liquid occurs. The cavity first grows, then collapses which means that the vapour phase turns back into liquid. The objective of the present numerical exercise was to try to model the process of such a latent energy being stored in the fluid medium (creation of liquid vapor), and later released back into fluid mechanical and kinetic energy [4]. For that very specific purpose the liquid cavitation produced by the projectile was modelled as a moving heat energy source that progressively produces water vapor in the liquid medium. No air was modelled (as it should be in the real problem, when it is trapped from the outside into the wake of the projectile), and no acoustic shock pressure was considered (since no solid projectile is modelled). These aspects are currently studied in parallel research works.

The first part of the present paper describes the EUROPLEXUS explicit code and general modelling philosophy. Then the numerical model and simulation results are presented and analysed, and the final conclusions and perspectives of the work are given. 


\section{Presentation of the EUROPLEXUS tool and modelling methodology}

\subsection{EUROPLEXUS general presentation}

EUROPLEXUS [5] is a computer code being jointly developed since 1999 by the French CEA and EC JRC Ispra. It stems from CEA's CASTEM-PLEXUS and the previous CEAEC joint product PLEXIS-3C. ONERA is member of the developing partners, for aeronautical applications. The program uses an explicit integration scheme (central-difference) for the discretization in time and therefore is best adapted to dynamic simulations (fast transient dynamics) such as explosions, impacts, etc. Geometric (large displacements and rotations, large strains), and material (plasticity, viscoplasticity, etc) non-linearities are fully taken into account. The code provides 1-D, 2-D or 3-D solid (continua, shells or beams) and fluid elements, with fluid/structure interaction functionalities being also implemented. Numerous element types and a comprehensive library of E.O.S and material laws for fluids, solids and special media (e.g. impedances) are available. Three main descriptions frameworks are available in the code: the Lagrangian description (including Smooth Particle Hydrodynamics) which is well suited for the structural domain, the Eulerian description for purely fluid problems, and the Arbitrary Lagrangian Eulerian (A.L.E.) description which is typically used in fluid-structure interaction problems.

\subsection{Finite volume formulation and Arbitrary Euler-Lagrange (ALE) description}

For numerical analysis, the Finite Volume Method (FV) is used to solve partial differential equations, like the finite element method (FE), where approximations of integrals are made. The FE method uses a variational formulation of the equations (or weak form) while the FV method is based on the direct form of the equations (strong form), that makes Navier-Stokes conservation equations solving possible. Finally, one has to solve the partial differential equations on a spatial discretization of the studied domain. A lot of information about the FV method can be found in the literature [6].

The ALE method allows an intermediate description of the studied domain to be used, that stands between purely Lagrangian mesh and purely Eulerian grid and makes the FV formulation applicable. It allows us to handle fluid-structure interactions with deformation of the fluid mesh that can adapt to the structural one, where a purely Eulerian one would not succeed. A more detailed presentation of the principles of this method, then the equation of conservation of a continuum medium and their resolution in the ALE frame are given in [7].

\subsection{Water equation of state and biphasic mixture law}

The objective is to run a 10 to $30 \mathrm{~ms}$ event simulation, in order to observe both the growing and collapsing of a vapour cavity. The difficulty of having a realistic simulation of the cavitation without having a biphasic law with change of state 
was pointed out by former simulations $[1,9]$. It was then decided to work with a V.F. method. As far as energy quantities are concerned, having an energy conservative formulation is preferred so as not to dissipate them numerically.

EUROPLEXUS allows handling water and its vapour as a homogeneous mixture [5], under its FV formulation. It is also possible to model a water vapour explosion when energy (e.g. explosion) is released in liquid water. Beside the biphasic mixture law, EUROPLEXUS provides E.O.S. to model water change of state, which includes the latent energy in the energy balance for higher accuracy. As water and its vapour are treated as a homogenous mixture, the tension energy of the gas-liquid interface is not considered. The tension energy is an important quantity for the understanding of the physics of the equilibrium and motion of bubbles in a quasi-static frame. In the present case, the tension energy can be neglected since it is small compared to the latent energy in the cavity (the ratio between the tension energy and the latent energy of the bubble is proportional to $\mathrm{R}^{-1}$, the inverse of the radius of the bubble, and we are studying large cavities here). The following considerations are made to model the problem:

- the water is in equilibrium, i.e. same pressure and same temperature for water and vapour within a fluid volume,

- the initial conditions of the bi-phasic material in sub-domains are given by an initial pressure (in $\mathrm{Pa}$, the reference pressure is ), an initial temperature (in $\mathrm{C}^{\circ}$ ) and an initial mass title (ratio between the mass of vapour and the mass of water, adimensional number),

- a time dependent specific energy is injected in the material (at the sources location), which has been chosen to be independent from the mass and nature (liquid or vapour) of the water. Thus, the injected energy is a volumic energy. One declared yet a specific energy in $\mathrm{J} / \mathrm{kg}$. The initial volumic mass of the material is used to convert this specific energy in volumic energy.

- a quite high numerical damping coefficient is used to increase the stability of the code by filtering high frequencies, at the expenses of amount of numerically dissipated energy,

The recorded output of the calculations are the output pressure, the density of the mixture, the sound speed, the mass title and volume ratio of vapour, the temperature of the mixture, the enthalpy, the injected power in the elements.

The physical properties of water are tabulated from the well known P, T diagram used to determine the title of the water (above $800^{\circ} \mathrm{C}$ ) [8]. Intervals are given to separate the low-temperature, the saturation curve, the high-temperature and the hypercritic domains of the $\mathrm{P}, \mathrm{T}$ diagram. This description greatly influences the complexity of the computations, since, for each time step, for each element, the algorithm must explore this diagram to determine the state of the water in the element.

\subsection{Cavitation source modelling}

The idea is now to model cavitation sources that develop along the projectile trajectory, and then to study the later expansion and collapsing of the vapor 
bubble, if ever. The displacement $\mathrm{d}(\mathrm{t})$ and speed $\mathrm{v}(\mathrm{t})$ of the $7,62 \mathrm{~mm}$ projectile in a long enough water pool has been recorded in [2]. As a starting point, we took that the cavitation production (creation of latent energy) is proportional to the loss of kinetic energy. Other quantities, like the pressure around the bullet or the velocity field of the fluid are hardly known with the reported experiments, then kinetic energy was the most reliable and only available energetic quantity to be used. Then, the power loss $\mathrm{W}(\mathrm{t})$ can be calculated at position $\mathrm{d}(\mathrm{t})$, at time $\mathrm{t}$, along the projectile trajectory. Function $\mathrm{d}(\mathrm{t})$ being an isomorphism, one can also write that a lineic energy $f(z)$ is delivered at position $\mathrm{z}$, at time $\mathrm{t}(\mathrm{z})$, which leads to formulate the energy loading function as a continuum of synchronised sources. The continuous model is then discretized for the numerical study, with $\mathrm{N}$ sources of coordinate $\mathrm{z}_{\mathrm{i}}$, defined here at regular $5 \mathrm{~mm}$ intervals and corresponding to the FV elements of the mesh along the projectile trajectory.

Finally, sources $S_{i}$ are active at time $t_{i}=t\left(z_{i}\right)$ and the injected energy $f_{i}=f\left(t_{i}\right)=f\left(z_{i}\right)$ is computed so that energy $k \Delta E_{c i}$ is delivered to the fluid, with:

$$
\Delta E_{c i}=\frac{E_{c i+1}-E_{i-1}}{2}
$$

with $\mathrm{E}_{\mathrm{i}}$ being the interpolated kinetic energy of the bullet at positions $\mathrm{z}_{\mathrm{i}}$. To avoid load discontinuities that can always generate numerical artefacts, the $f_{i}$ injection functions are given a triangular shape between $t_{i-1}$ and ${ }_{t+1}$, and an $f_{i \max }$ maximum amplitude, that gives the expected $k \Delta E_{c i}$ injected energy once integrated.

\subsection{Energy injection method}

An injected mass (of fluid) method would consist in injecting mass in the FV sources of volume Vi of boundary surfaces $\mathrm{S}_{\mathrm{Vi}}$, then generating mass flux through the mesh. This way of injecting energy sounds like a good approximation of the real case - as long as the injected mass is small enough compared to the total mass of fluid - because it leads to a liquid mass flux like the projectile which moves in the liquid, and can be related to the kinetic energy transfer from the projectile to the fluid as follows:

$$
\dot{m}_{i}(t)=\sqrt[3]{2 \cdot\left(\rho \cdot S_{V i}\right)^{2} \cdot \alpha \Delta E_{c_{i}} \cdot f_{i}(t)}
$$

Unfortunately, this kind of energy injection method is not available in EUROPLEXUS without specific developments. A second method would of course consist in direct injection of energy in the model. Such a method is possible in EUROPLEXUS, with the energy being injected as a heat that increases the specific enthalpy $h$ of water in the FV source $S_{i}$ (latent heat is a particular form of enthalpy for water). It initiates local ebullition then cavitation, as reported by the Ecole Polytechnique Federal de Lauzanne in [10]. The 
principle is presented in fig. 3. In the real case, the bullet induces a radial displacement of the water. Because of the depression, water is vaporized. If one writes the energy balance at the interface between water and vapour, neglecting compression of water and viscous forces, the following equation is obtained:

$$
W_{p}=L \cdot \dot{m}
$$

where $\mathrm{W}_{\mathrm{p}}$ denotes the work of the pressure forces, $\mathrm{L}$ is the latent heat of the vapour, and $\dot{m}$ the quantity of water which is vaporized at the interface. No variation of temperature in vapour phase needs to be considered (at saturation pressure and temperature in the test). By integrating the above equation, a conversion mechanism of projectile kinetic energy into vapour latent heat is modelled (see fig. 3). During the collapse of the cavity, the contrary phenomenon occurs.

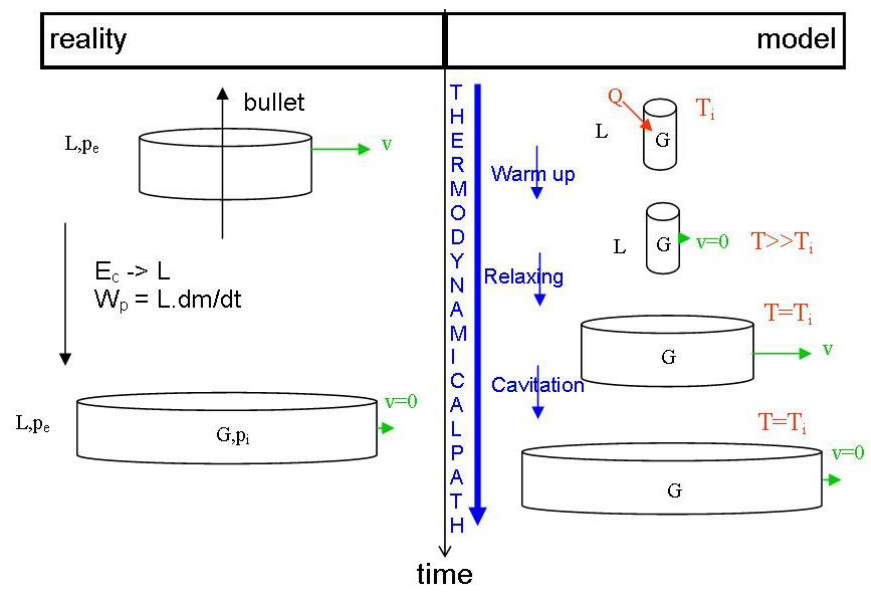

Figure 3: Simplified principle of the energy injection method by heat.

By heating the sources volume, water is vaporised and overheated. The vapour is in compression state, then relaxes (in an anisotropic way) which produces kinetic energy through the surrounding water. Later on, when the vapour has relaxed, the water has accumulated enough kinetic energy to induce cavity expansion with its own cavitation production. A new equilibrium is then reached, that leads to the cavity collapse. An energy restitution coefficient $\alpha$ is defined as the ratio between the mechanical energy (kinetic energy, potential energy of the pressure forces) given to the system by the projectile and the injected heat. Since the vaporization process (heat and relax) is very fast, an adiabatic assumption is made, which means no heat transfer between the vapour created in the FV sources and the water in the surrounding elements, hence no vaporization of water but the one initially present in the given FV sources. So, the energy used to warm the water up to $100^{\circ} \mathrm{C}$ and vaporize it in the $\mathrm{FV}$ sources 
becomes smaller as the volume of the sources decreases, and most of the injected energy is used to warm up the vapour which relaxes in an adiabatic way. If one assumes that the vapour is a Laplace gas, the following equations are obtained for the potential energy of the pressure forces, the injected latent heat, and the energy restitution coefficient:

$$
p \cdot V=\left(1-\frac{1}{\gamma}\right) \cdot h, L=\alpha k \Delta E_{c i}, \alpha=\gamma /(\gamma-1)
$$

Since not all of the injected energy is converted into mechanical energy, an elevation of temperature is observed after the relaxation phase. If this elevation is small enough, one would assume that the physics of the water in the tank is not too different from the one in the real case.

\section{Numerical results}

In the presented simulation, a Au2024 T321 metallic dummy tank with $6 \mathrm{~mm}$ thick aluminum top and bottom skins that was studied in the EUCLID RTP3.32 project is studied. The transparent windows are made of $50 \mathrm{~mm}$ thick PMMA material. The aluminum material is modelled using a perfect elasto-plastic material model with a Von Mises criterion, with: $E=66 \mathrm{Gpa}, \rho=2,6.10^{+3} \mathrm{~kg} /$ $\mathrm{m}^{-3}, \sigma_{v}=200 \mathrm{Mpa}, v=0,31$, where $\mathrm{E}$ is the young Modulus, $\rho$ the material density, $\sigma_{y}$ the elastic yield stress, and $v$ the Poisson ratio.

The $300 \times 460 \times 540 \mathrm{~mm}^{3}$ fluid volume is modelled using the EUROPLEXUS water equation of state and bi-phasic mixture law [5], with the $[0,6000]^{\circ} \mathrm{C}$ temperature and $\left[7.10^{-3}, 3.10^{+4}\right]$ bar pressure intervals for the $\mathrm{P}, \mathrm{T}$ diagram being split into 80 intervals for the low temperature domain, 150 intervals for the saturation curve, 150 intervals for the high temperature domain, and 180 intervals for the hypercritic domain. For the energy injection model, a $\mathrm{k}=1$ ratio (with respect to the $3 \mathrm{~kJ}$ projectile initial kinetic energy) was taken to maximise the cavitation production in the simulation, and a water thermo-dynamical table was used to choose a $\gamma=7 / 5=1,4$ value, which gives a $\alpha=3,5$ value for the correction factor. Since water is a three atoms molecule, one should expect $1<\gamma<7 / 5$, then a correction factor higher than 3,5: the loading of the structure in the present simulations is then underestimated.

The F.V-FE mesh is volumic for both the fluid and the structure domains. Adopting a volumic mesh for the structure is not penalizing since the volume of water is 30 times bigger than the one of the structure. The FV sources are placed along the $\mathrm{Oz}$ axis. A conform mesh is used, i.e. the nodes of the solid elements and the nodes of the fluid volumes are coincident at the interfaces. For such a mesh, the transmission of forces is automatically handled by the code and no specific declaration or use of Lagrangian multiplicators to solve fluid/structure interactions is needed. Conform meshing improves the stability of the code and greatly increases the precision in the determination of local volume changes. To get a good flexion of the structure walls, the thickness of the solid elements is 
fixed to $1 \mathrm{~mm}$ (6 elements through the skins thickness). The length of the fluid volumes along the projectile trajectory is constant and is $5 \mathrm{~mm}$ large to get an affordable simulation time. Such a length allows us to set on 56 heat sources in the water along the projectile trajectory. In the vicinity of the projectile trajectory, the length of finite elements and volumes along $\mathrm{x}$ and $\mathrm{y}$ is $1 \mathrm{~mm}$ (to minimise the volume of the sources and minimise the energy lost in prewarming), increasing linearly to $10 \mathrm{~mm}$ at the structural side walls. Finally, the mesh is 130000 finite elements and 576288 finite volumes large.

The results were analysed at several time scales: during the first millisecond of the energy injection-convection phase, and during a longer eight milliseconds timespan. Among various exploited data, the pressure field, temperature and volumic title in the fluid are illustrated hereafter. The pressure and temperature are plotted with logarithmic scales $\left(50 \mathrm{bars}\right.$, and $4500^{\circ} \mathrm{C}$, respectively) to cover large gradients during the loading and convecting steps. The vapour volume title is plotted using a linear $0-1$ scale, to picture the caviting step.

The maximum temperature value during the loading phase of one source was about a few hundred degrees. This temperature increases in some of the FV sources up to a few thousands degrees (e.g. between 0,05 ms and 0,25 ms) after this sourcing phase: this can be explained because of convection process in the vapor cavity. Then, the temperature of the vapor decreases rapidly: after one millisecond, it has get down to $60^{\circ} \mathrm{C}$, and $30^{\circ} \mathrm{C}$ after eight milliseconds, which is far below the saturation temperature at 1 bar.

The maximum peak pressure at $\mathrm{t}=0,2 \mathrm{~ms}$ and $5 \mathrm{~cm}$ from the shot line in the simulations is about 5 bars (see fig. 4 and 6), then largely (one order of magnitude) below the 50 bars measured in the tests [1, 2, 4]. This can be explained because the mechanical shock between the projectile and water medium is not modelled in this simplified exercise.
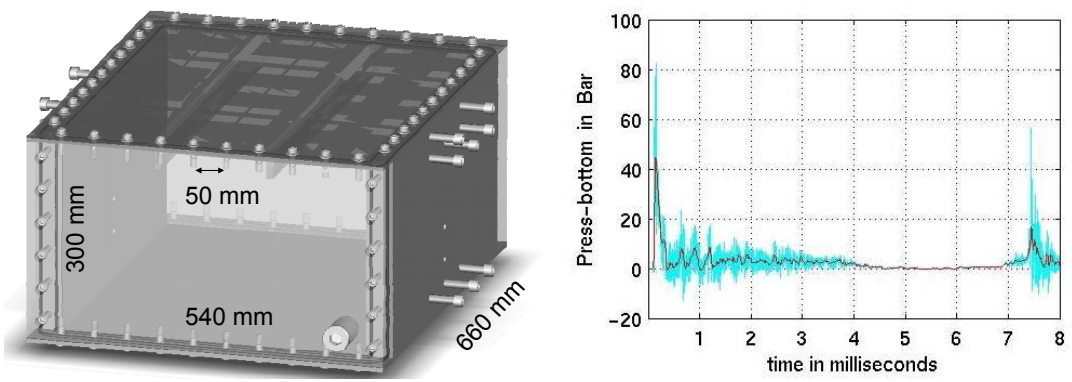

Figure 4: Sketch of the EADS-IWF dummy boxes geometry (left) and pressure measurement $15 \mathrm{~cm}$ away from the shotline during test (right). 


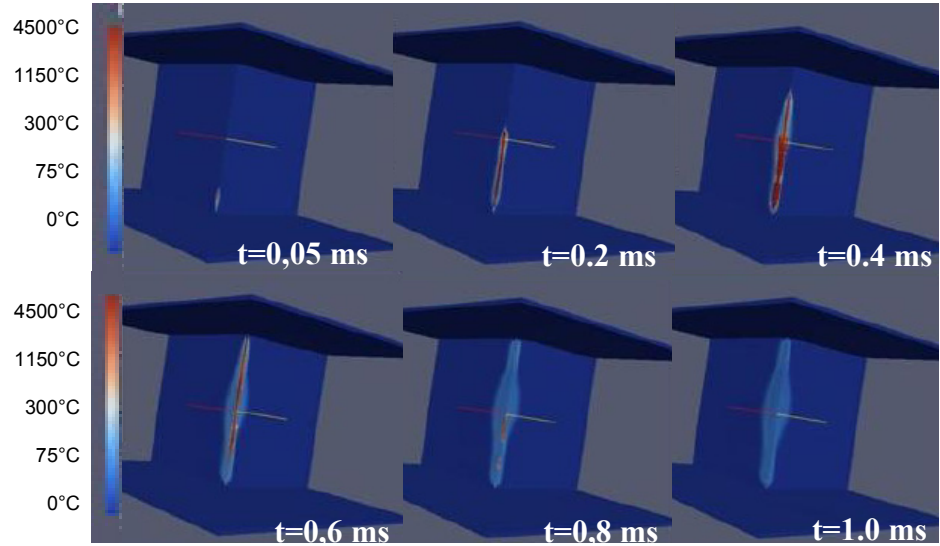

Figure 5: $\quad$ Heat injection-convection $1 \mathrm{~ms}$ phase $\left(0-4500^{\circ} \mathrm{C} \log\right.$. scale).

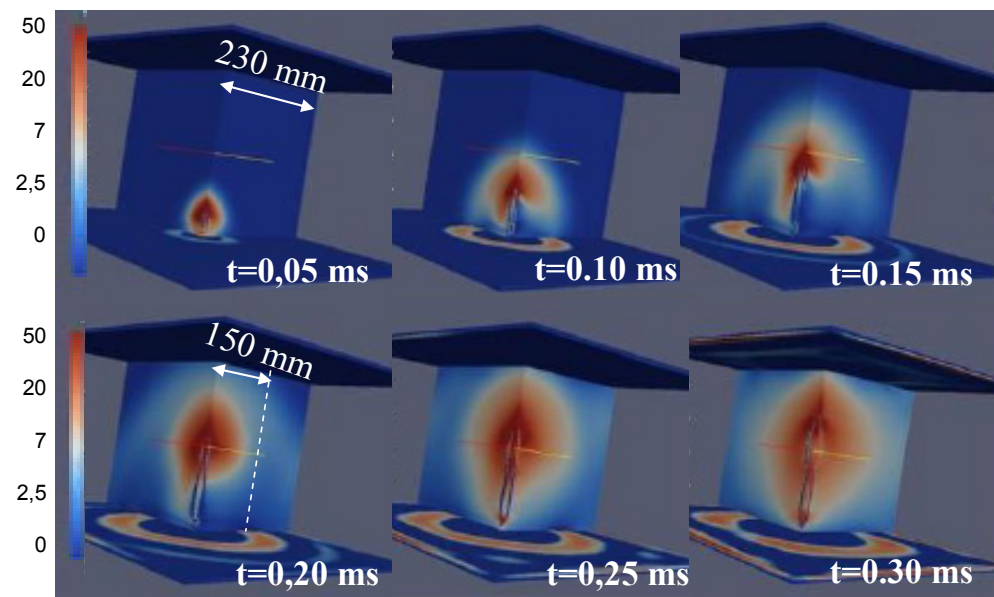

Figure 6: $\quad$ Loading phase $0-0,3 \mathrm{~ms}(0-50$ bars log. scale).

The lifetime of the cavity is $7,41 \mathrm{~ms}$ in the simulation, which correlates well with the 7, $5 \mathrm{~ms}$ one observed in the EUCLID RTP3.32 test. But the obtained size and shape of the cavity in the numerical simulations turn to be a little more different from the experimental observed ones (in pool or box): the maximum radius of the cavity is about $60 \mathrm{~mm}$ in the simulation where it was $75 \mathrm{~mm}$ in the box experiments. This is due to the previously described convection process that very quickly homogenizes the internal energy in the vapor phase, which means that the activated downstream sources re-energize the upstream FV elements. The expansion of the vapor cavity between $1 \mathrm{~ms}$ and $8 \mathrm{~ms}$ should be governed by mechanical cavitation dynamics, but the heat convection keeps the corresponding sources active when these should be "shut": the cavity keeps on expending in areas by thermal effects where and when it should only be by mechanical ones. 


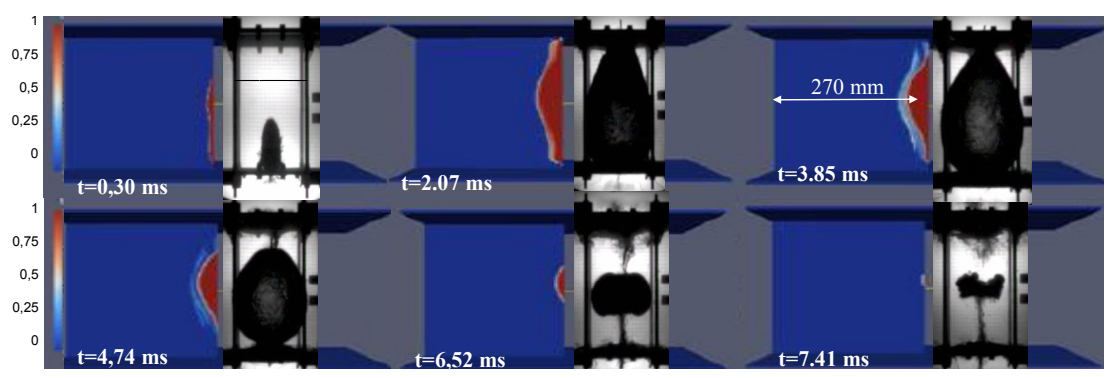

Figure 7: Cavity dynamics phase $0,3-7,5 \mathrm{~ms}$ (vapour volume title - 0-1 scale).

\section{Conclusions and perspectives}

The numerical simulation of ballistic impacts into fuel tanks raises many difficulties because of the highly dynamic and non-linear phenomena that develop during Hydraulic Ram events. In particular, the prediction of both the high pressure shock levels and the air-vapour cavity volume in the wake of the projectile is needed to be able to mechanically design and size such structures. Complex fluid change of state and fluid/structure coupling issues need then to be solved, which is a broad research challenge. The present paper focuses on the question of modelling the growth and collapsing phase of liquid vapour made cavities that develop in the wake of high speed projectiles, due to cavitation (i.e. change of state) mechanisms. An energy injection based method is proposed, together with the use of a mixed FV/FE EUROPLEXUS model, in order to study hydrodynamic effects like the ones expected in HRAM events. The injected energy is calculated with respect to the variation of kinetic energy of such a projectile when it travels through the fluid, which was measured in previous research works. Then equivalent latent heat is progressively loaded into the system, which corresponds to this energy injection rate. With the proposed methodology and model, it was possible to successfully study a cavity growth/collapsing phenomenon similar to the one observed during HRAM tests, with a satisfactory CPU time (5 days to simulate the $8 \mathrm{~ms}$ full collapse of the cavity). But this achievement was at the price of simplifications that led, for instance, to loss of anisotropy of the loading (due to relaxation time of vapour), that is suspected to induce mechanical shock softening in the modelling.

The perspectives of this research are, first, to improve the water change of state physical model with a possible second gas being considered in the mixture law (air trapped in the projectile wake). Water being not what makes aircraft fly, real fuel should also be studied. Second, a full 3D simulation exercise of a soft/highly deformable projectile penetrating a liquid medium is in progress, in order to try to develop numerical solutions that can cope with such complex and difficult fluid/structure interaction situations (Smooth Particle Hydrodynamics, Euler/Lagrange coupling methods, etc, are investigated). Last, but not least, highly sophisticated experimental tests with heavy dynamic instrumentation have 
to be further developed, to be able to validate the new physical model or numerical solver that will be proposed.

\section{Acknowledgement}

The presented work is based on test results that were obtained with the financial support of the French Delegate for Armament (DGA/SPAé) in the frame of the European EUCLID RTP3.32 program under the aegis of EDA.

\section{References}

[1] Deletombe E., Fabis J. and Dupas J., Resistance of composite materials and tank structures to the impact and hydraulic ram pressure generated by a ballistic projectile. Proc. of DYMAT Conference, eds. EDP Sciences, 2009.

[2] Deletombe E., Fabis J., Dupas J. and Mortier J.-M., Experimental analysis of $7.62 \mathrm{~mm}$ hydrodynamic ram in containers. Journal of Fluid and Structures (in press).

[3] Ankeney D. B., Physical Vulnerability of Aircraft due to Fluid Dynamic Effects. AGARD Advisory Report, n¹06, 1977.

[4] Deletombe E., Fabis J. and Dupas J., Vulnerability of A/C fuel tanks with respect to hydrodynamic ram, pressure - Interpretation of 7, $62 \mathrm{~mm}$ Experiments. Proc. of Colloque National en Calcul des Structures (CSMA), 2010.

[5] CEA, CEC ISPRA, EUROPLEXUS User's manual.

[6] Stoukov A., Introduction to fluid mechanics: finite volume method, online lecture, http://hmf.enseeiht.fr/coursenligne/stoukov/vf.pdf

[7] Donea J. et al., Arbitrary Lagrangian-Eulerian Methods (Chapter 14). Encyclopaedia of Computational Mechanics, Encyclopedia of Computational Mechanics, ed. John Wiley \& Sons, Ltd., 2004.

[8] Properties of Water and Steam in SI-Units, ed. Springer Berlin Heidelberg, 1979.

[9] Lecysyn N. et al., Experimental study of hydraulic ram effects on a liquid storage tank: Analysis of overpressure and cavitation induced by a highspeed projectile. Journal of hazardous materials, 178 (no. 1-3), pp. 635643, 2010.

[10] A. de Bosset et al, Direct Effects of Gravity on Cavitation Bubble Collapse. Proc. of the 58th International Astronautical Congress, 2007. 